CLINICAL STUDY

\title{
Serum calcium level is associated with metabolic syndrome in the general population: FIN-D2D study
}

\author{
J Saltevo $^{1}$, Leo Niskanen ${ }^{1,2}$, Hannu Kautiainen ${ }^{3,4}$, Jorma Teittinen ${ }^{5}$, Heikki Oksa ${ }^{6}$, Eeva Korpi-Hyövälti ${ }^{7}$, \\ Jouko Sundvall $^{8}$, Satu Männistö ${ }^{9}$, Markku Peltonen ${ }^{10}$, Pekka Mäntyselkä ${ }^{4}$ and Mauno Vanhala ${ }^{3,4}$ \\ ${ }^{1}$ Department of Medicine, Central Finland Central Hospital, 40620 Jyväskylä, Finland, ${ }^{2}$ Faculty of Health Sciences, University of Eastern Finland, Kuopio, \\ Finland, ${ }^{3}$ Unit of Family Practice of Central Finland Central Hospital, Jyväskylä, ${ }^{4}$ Unit of Primary Health Care, University of Eastern Finland, and Kuopio \\ University Hospital, Kuopio, Finland, ${ }^{5}$ Central Finland Central Hospital, Jyväskylä, Finland, ${ }^{6}$ Tampere University Hospital, Tampere, Finland, ${ }^{7}$ Department \\ of Internal Medicine, South Ostrobothnia Hospital District, Seinäjoki, Finland, ${ }^{8}$ Disease Risk Unit, National Institute for Health and Welfare, Helsinki, \\ Finland, ${ }^{9}$ Chronic Disease Epidemiology and Prevention Unit, National Institute for Health and Welfare, Helsinki, Finland and ${ }^{10}$ Diabetes Prevention Unit, \\ National Institute for Health and Welfare, Helsinki, Finland
}

(Correspondence should be addressed to J Saltevo; Email: juha.saltevo@ksshp.fi)

\begin{abstract}
Background: The aim of this cross-sectional study was to examine the association between serum calcium and the components of metabolic syndrome (MetS).

Methods: As a part of the national prevention program of diabetes in Finland (FIN-D2D), a randomly selected study population of 4500 middle-aged men and women were recruited from three central hospital district areas. Anthropometric measurements were performed by a trained nurse. An oral glucose tolerance test was performed and serum calcium and lipids were measured. We assessed current medications, physical activity, smoking, alcohol consumption, calcium intake, and vitamin D intake. The MetS was defined according to the criteria of the updated National Education Program. The study population consisted of 2896 individuals: 1396 men (62\% of invited individuals) and 1500 women $(66.7 \%$ of invited individuals).

Results: The mean age was $60.3 \pm 8.3$ years in men and $59.8 \pm 8.5$ years in women. The prevalence of MetS was $50.7 \%$ in women and $55.8 \%$ in men. The prevalence of MetS and its components, except high-density lipoprotein (HDL)-cholesterol, increased linearly with increasing serum calcium $(P<0.001)$, even after adjustment for age, physical activity, alcohol, vitamin $\mathrm{D}$ intake, calcium intake, and smoking. The threshold value for serum calcium for MetS was $2.50 \mathrm{mmol} / \mathrm{l}$ in this population. The association of MetS with total serum calcium was similar even after exclusion of patients treated with hypertensive drugs. The drug treatments for hypertension, dyslipidemia, and diabetes increased in a similar pattern.

Conclusions: Serum calcium level is associated with MetS and its components, except HDL-cholesterol.
\end{abstract}

European Journal of Endocrinology 165 429-434

\section{Introduction}

Metabolic syndrome (MetS) is a well-known clustering of multiple cardiovascular risk factors in an individual. The constellation of metabolic abnormalities includes glucose intolerance, insulin resistance, central obesity, dyslipidemia, high triglyceride levels, low high-density (high-density lipoprotein (HDL))-cholesterol levels, and hypertension. All of these variables are well-documented risk factors for cardiovascular disease (CVD). These conditions co-occur in an individual with MetS more often than what could be expected by chance, and they are associated with an increased risk for CVD (1) and type 2 diabetes (2).

Previous reports have shown that patients with diabetes have elevated serum calcium levels compared with non-diabetic individuals (3), and this has mainly been linked to impaired insulin sensitivity rather than defective insulin secretion (4). In diseases with markedly elevated serum calcium, such as primary hyperparathyroidism, there is a two- to four-fold higher prevalence of type 2 diabetes and glucose intolerance compared with the general population (5). Increased parathyroid hormone (PTH) could stimulate calcium channels to increase calcium influx and the levels of intracellular calcium, which would influence insulin sensitivity and hypertension (6). Increasing intracellular calcium levels have been shown to decrease the effect of insulin in adipocytes due to reduced number of glucose transporters (GLUT4) and decreased insulin receptor activity $(5,7)$. The dietary intake of calcium did not seem to influence insulin sensitivity (4).

A Swedish 18-year follow-up study found a positive association between all causes of mortality and high 
levels of serum calcium, especially in men. Inclusion of the serum calcium level increased the predictive value of the traditional risk factors, which suggested an independent mediating mechanism (8).

Because serum calcium is connected with insulin sensitivity and CVDs, we hypothesized that calcium was associated with the precursor of type 2 diabetes and CVD, namely MetS. Thus, serum calcium would also be associated with the individual components of MetS. We explored this issue in a large population-based study.

\section{Methods}

\section{Participants and study design}

The FIN-D2D survey was carried out in the hospital districts of Pirkanmaa, the Southern Ostrobothnia, and Central Finland between October and December 2007. A random sample of 4500 subjects aged 45-74 years old, which was stratified according to gender, 10-year age groups (45-54, 55-64, and 65-74 years), and the three geographical areas, was selected from the National Population Register in August 2007. The study participants were invited to a clinical examination by mail. The overall participation rate was $64 \%$. Thus, the study included 2896 individuals: 1396 were men ( $62 \%$ of men invited) and 1500 were women $(66.7 \%$ of women invited).

The study protocol was approved by the ethics committee of the Hospital District of Helsinki and Uusimaa. All participants gave their written informed consent prior to participation in the study.

The health examination was carried out according to the World Health Organizations (WHO) MONICA project and the WHO Expert Group for glucose assessments. Food consumption over the previous 12 months was assessed with a validated self-administered food frequency questionnaire (FFQ). The participants were asked to estimate the consumption frequencies of 132 food items or mixed dishes listed on the FFQ using ninepoint scale ranging from 'never or seldom' to 'at least 6 times per day' (9). The portion sizes could be compared with the predefined sizes printed on the questionnaire. The food data were converted into nutrient intakes (e.g. calcium and vitamin D) using the software and the Finnish national food composition database, Fineli, at the National Institute for Health and Welfare.

Alcohol consumption during the past week and the past year was assessed with a self-administered questionnaire. The average daily alcohol consumption (g/day) was calculated from the number of drinks during the past week. The participants were also asked whether they were using lipid-lowering, antihypertensive, or antihyperglycemic medications.

Height, weight, and waist circumference were measured by nurses who were specially trained for the survey procedures. Height was measured to the nearest $0.1 \mathrm{~cm}$, and weight was measured to the nearest $0.1 \mathrm{~kg}$ in light clothing. Body mass index (BMI) was calculated as weight $(\mathrm{kg})$ divided by the height squared $\left(\mathrm{m}^{2}\right)$. Blood pressure (BP) was measured twice, and the latter was used in the analysis, in a sitting position after a minimum of 15 min of acclimatization using a mercury sphygmomanometer.

\section{Laboratory analysis}

After an overnight fast, venous blood samples were drawn into a gel tube (containing clot activator) for serum lipid, creatinine, and total calcium assays and a fluoride citrate tube (Venosafe, Terumo Europe, Leuven, Belgium) for the plasma glucose assay. The samples were immediately frozen after separating serum and plasma and transferred to the laboratory in dry ice once a week for analyses. An oral glucose tolerance test was performed in all subjects except for those with previously diagnosed diabetes (either type 1 or type 2 ).

All assays were performed at the Laboratory of Analytical Biochemistry at the National Public Health Institute, Helsinki (Disease Risk Unit, Institute for Health and Welfare since 2009), using an ARCHITECT ci8200 analyzer (Abbott Laboratories). The laboratory has been accredited by Finnish Accreditation Service (FINAS, Helsinki, Finland) and it fulfills the requirements of the standard SFS-EN ISO/IEC 17025:2005. The scope of accreditation (T077) covers all analyses. Serum concentrations of total cholesterol, HDL-cholesterol, triglycerides, and creatinine were measured with fully enzymatic assays using Abbott ARCHITECT reagents, except creatinine with reagents from Thermo Fisher Scientific (Vantaa, Finland). The glomerular filtration rate was calculated with the Cockcroft-Gault equation. Plasma glucose was determined with a hexokinase method (Abbott Laboratories), and serum total calcium was measured photometrically on an ARCHITECT ci8200 analyzer using an arsenazo III method (Abbott Laboratories).

To ensure standardization of biochemical measurements, the laboratory takes part in Lipid Standardization Program organized by the Centers for Disease Control (CDC, Atlanta, GA, USA) and External Quality Assessment Schemes organized by Labquality (Helsinki, Finland). During the course of the biochemical measurements comprising of 4 months, the precision values between series expressed as coefficient of variation for calcium at the levels $1.57,2.29$, and $3.13 \mathrm{mmol} / \mathrm{l}$ were $1.3,1.4$, and $1.1 \%$; for total cholesterol at the levels $4.9,5.5$, and $6.0 \mathrm{mmol} / \mathrm{l}$ were $0.8,0.8$, and $0.7 \%$; for HDL-cholesterol at the levels $0.89,1.22$, and $1.70 \mathrm{mmol} / \mathrm{l}$ were $2.8,2.0$, and $2.2 \%$; for triglycerides at the levels $0.96,1.25$, and $2.62 \mathrm{mmol} / \mathrm{l}$ were $1.2,1.2$, and $0.8 \%$; for glucose at the levels 3.4 , 5.6, and $20.4 \mathrm{mmol} / \mathrm{l}$ were $1.5,1.3$, and $1.3 \%$; and for creatinine at the levels 67,77 , and $579 \mu \mathrm{mol} / \mathrm{l}$ were $1.9,2.1$, and $1.5 \%$. The systematic error for different reference samples (mean bias \pm s.D.) 
was $-1.2 \% \pm 1.0(n=4)$ for calcium $0.7 \% \pm 0.6(n=8)$ for total cholesterol, $-0.1 \% \pm 1.6(n=8)$ for HDLcholesterol, $0.6 \% \pm 1.9 \quad(n=8)$ for triglycerides, $-0.7 \% \pm 1.4(n=4)$ for glucose, and $-0.9 \% \pm 1.6$ $(n=4)$ for creatinine.

\section{Definition of MetS}

MetS was defined according to criteria of the updated National Education Program (NCEP) Expert Panel on Detection, Evaluation, and Treatment of High Blood Cholesterol in Adults (ATP III) (10). Subjects with three or more of the following components were classified as having MetS: i) central obesity (waist circumference $>102 \mathrm{~cm}$ in men and $>88 \mathrm{~cm}$ in women); ii) elevated fasting serum triglycerides $(\geq 1.70 \mathrm{mmol} / \mathrm{l})$ or specific treatment for this lipid abnormality; iii) low serum HDLcholesterol $(<1.03 \mathrm{mmol} / \mathrm{l}$ in men and $<1.29 \mathrm{mmol} / \mathrm{l}$ in women) or specific treatment for this lipid abnormality; iv) systolic BP $\geq 130 \mathrm{mmHg}$, diastolic BP $\geq 85 \mathrm{mmHg}$ or treatment for previously diagnosed hypertension; or v) fasting plasma glucose $\geq 5.6 \mathrm{mmol} / \mathrm{l}$ or previously diagnosed type 2 diabetes.

\section{Statistical analysis}

The results are expressed as mean \pm s.D. The study population was divided into four groups according to the mean $(2.49 \mathrm{mmol} / \mathrm{l}) \pm$ s.D. $(0.1 \mathrm{mmol} / \mathrm{l})$ of serum total calcium values $(\leq 2.39,2.40-2.49,2.50-2.59$, $\geq 2.60 \mathrm{mmol} / \mathrm{l})$. Statistical significance for the hypotheses of linearity was evaluated by Bootstrap-type ANOVA and logistic regression models with covariates when appropriate. Sensitivity and specificity curves according to the range of serum total calcium with and without MetS are illustrated using a two-graph receiver operating characteristic (ROC). The ROC curves were used for determination of thresholds for the MetS group compared to the group without MetS, and the respective areas under the curve (AUC) were calculated with a biascorrected accelerated bootstrap confidence interval (CI). The threshold values of plasma serum calcium level with the highest accuracy (sum of sensitivity and specificity) separating subjects with and without MetS were determined from an ROC curve. Differences between the AUC were evaluated using an algorithm by DeLong. STATA 11.1, StataCorp LP (College Station, TX, USA) statistical package was used for the analyses.

Table 1 Basic characteristics of study population according to the serum total calcium (mmol/l). Data are mean (S.D.).

\begin{tabular}{|c|c|c|c|c|c|}
\hline & \multicolumn{4}{|c|}{ Serum calcium $(\mathrm{mmol} / \mathrm{l})$} & \multirow[b]{2}{*}{$P$ value } \\
\hline & $\leq 2.39$ & $2.40-2.49$ & $2.50-2.59$ & $\geq 2.60$ & \\
\hline \multicolumn{6}{|l|}{ Men } \\
\hline$n$ & 154 & 606 & 487 & 97 & \\
\hline Age & $62(8)$ & $60(9)$ & $60(8)$ & $61(8)$ & 0.28 \\
\hline $\mathrm{BMl}\left(\mathrm{kg} / \mathrm{m}^{2}\right)$ & $27.2(3.9)$ & $27.4(4.5)$ & $27.5(4.0)$ & $28.1(3.7)$ & 0.015 \\
\hline Waist $(\mathrm{cm})$ & 99 (12) & $100(12)$ & $100(11)$ & $103(11)$ & 0.044 \\
\hline Systolic blood pressure $(\mathrm{mmHg})$ & 137 (19) & $136(18)$ & $141(19)$ & 141 (19) & 0.001 \\
\hline Diastolic blood pressure (mmHg) & $82(11)$ & $82(10)$ & $84(10)$ & $85(9)$ & 0.003 \\
\hline Fasting plasma glucose (mmol/l) & $6.2(0.9)$ & $6.4(1.2)$ & $6.5(1.3)$ & $6.7(1.8)$ & $<0.001$ \\
\hline $2 \mathrm{~h}$ plasma glucose $(\mathrm{mmol} / \mathrm{l})$ & $6.8(2.5)$ & $7.0(3.0)$ & $7.4(2.9)$ & $8.2(4.8)$ & $<0.001$ \\
\hline Total cholesterol $(\mathrm{mmol} / \mathrm{l})$ & $5.0(1.0)$ & $5.3(1.0)$ & $5.5(1.0)$ & $5.4(1.1)$ & $<0.001$ \\
\hline HDL-cholesterol (mmol/l) & $1.27(0.30)$ & $1.31(0.31)$ & $1.34(0.33)$ & $1.30(0.32)$ & 0.11 \\
\hline Triglycerides $(\mathrm{mmol} / \mathrm{l})$ & $1.35(0.67)$ & $1.40(0.85)$ & $1.58(1.17)$ & $1.80(1.16)$ & $<0.001$ \\
\hline S-GFR (ml/min) & $95(28)$ & $94(25)$ & $94(24)$ & $94(21)$ & 0.98 \\
\hline Dietary calcium (mg/day) & $1463(628)$ & $1508(685)$ & $1520(681)$ & 1760 (939) & $<0.007$ \\
\hline Dietary vitamin D ( $\mu \mathrm{g} /$ day $)$ & $11.0(7.0)$ & $11.1(6.2)$ & $11.5(6.8)$ & $13.5(17.2)$ & 0.023 \\
\hline \multicolumn{6}{|l|}{ Women } \\
\hline$n$ & 183 & 625 & 536 & 138 & \\
\hline Age & $54(9)$ & $58(8)$ & $61(8)$ & $62(7)$ & $<0.001$ \\
\hline $\mathrm{BMl}\left(\mathrm{kg} / \mathrm{m}^{2}\right)$ & $26.5(6.0)$ & $27.4(5.1)$ & $27.8(5.2)$ & $28.9(5.6)$ & $<0.001$ \\
\hline Waist (cm) & $88(14)$ & $90(13)$ & $91(13)$ & $94(14)$ & $<0.001$ \\
\hline Systolic blood pressure $(\mathrm{mmHg})$ & $128(19)$ & $135(19)$ & $137(19)$ & $141(17)$ & $<0.001$ \\
\hline Diastolic blood pressure $(\mathrm{mmHg})$ & $78(9)$ & $81(9)$ & $80(8)$ & $81(9)$ & 0.004 \\
\hline Fasting plasma glucose (mmol/l) & $5.9(1.3)$ & $6.0(1.0)$ & $6.1(1.0)$ & $6.2(1.0)$ & $<0.001$ \\
\hline $2 \mathrm{~h}$ plasma glucose $(\mathrm{mmol} / \mathrm{l})$ & $6.5(2.3)$ & $6.8(2.2)$ & $7.3(2.5)$ & $7.5(2.6)$ & $<0.001$ \\
\hline Total cholesterol (mmol/l) & $5.2(0.8)$ & $5.5(0.9)$ & $5.7(1.0)$ & $5.7(1.1)$ & $<0.001$ \\
\hline HDL-cholesterol (mmol/l) & $1.52(0.34)$ & $1.54(0.33)$ & $1.56(0.33)$ & $1.58(0.37)$ & 0.12 \\
\hline Triglycerides (mmol/l) & $1.12(0.60)$ & $1.22(0.57)$ & $1.36(0.63)$ & $1.44(0.65)$ & $<0.001$ \\
\hline S-GFR (ml/min) & $88(27)$ & $83(23)$ & $80(23)$ & $81(22)$ & 0.003 \\
\hline Dietary calcium (mg/day) & $1518(683)$ & $1456(587)$ & 1475 (632) & $1563(660)$ & 0.55 \\
\hline Dietary vitamin D ( $\mu \mathrm{g} /$ day $)$ & $8.2(4.0)$ & $8.3(4.3)$ & $9.0(5.2)$ & $9.0(3.9)$ & 0.01 \\
\hline
\end{tabular}

*Significance test for linear trend. GFR, glomerular filtration rate. 


\section{Results}

The mean ages of the men and the women were 60.3 \pm 8.3 and $59.8 \pm 8.5$ years old respectively. The BMI was $27.7 \pm 4.1 \mathrm{~kg} / \mathrm{m}^{2}$ for men and $27.7 \pm 5.2 \mathrm{~kg} / \mathrm{m}^{2}$ for women. Table 1 shows that all components of the MetS - except HDL-cholesterol levels - linearly increased with the calcium in both genders. There was a linear trend with increasing calcium and age in women $(P<0.001)$, but not in men $(P=0.28)$. In men, the daily dietary intake of calcium $(P<0.007)$ and vitamin $D$ $(P=0.023)$ increased linearly with the serum calcium. However, in women, only dietary intake of vitamin D was statistically significant $(P=0.01)$. In women, serum glomerular filtration rate decreased linearly with increasing serum total calcium values $(P=0.003)$, but not in men $(P=0.98)$. This association was seen in women after excluding hypertensive patients $(P<0.001)$, but not in men $(P=0.64)$.

In predicting MetS, the sensitivity and specificity curves according to the range of values of serum calcium values are illustrated in Fig. 1B. Areas under the ROC curve were 0.56 (95\% CI: 0.54-0.58) for all subjects, $0.58(0.55-0.61)$ in women and 0.54 (0.51-0.57) in men. There was no statistical difference between genders. The threshold level of serum calcium for MetS was $2.50 \mathrm{mmol} / \mathrm{l}$. Gender and weight-adjusted area under ROC was 0.55 (95\% CI: 0.52-0.59).

The prevalence of the updated NCEP-MetS was $50.7 \%$ in women and $55.8 \%$ in men. It increased according to the serum calcium in a linear trend $(P<0.001)$, even after adjustment for age, physical activity, alcohol intake, dietary intake of vitamin $\mathrm{D}$, and smoking (Fig. 1). The drug treatment for hypertension increased linearly in both genders $(P=0.005$ for men and $P<0.001$ for women). The same linear trend was also seen in the drug treatment for dyslipidemia and diabetes in men and women (Table 2).
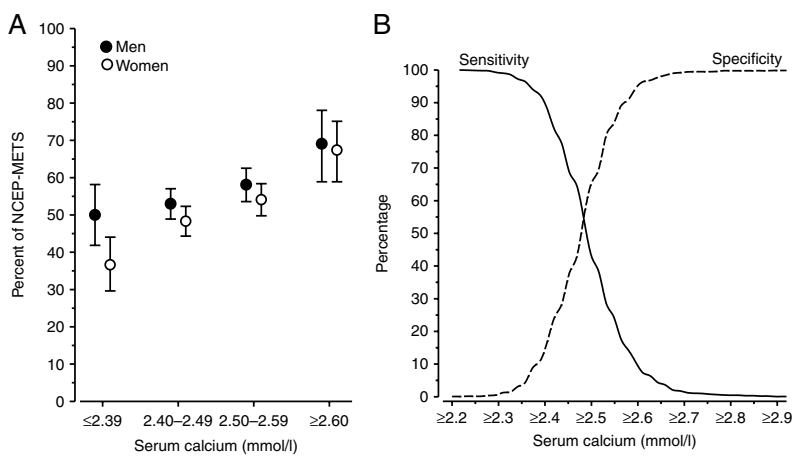

Figure $1(A)$ The prevalence of the updated NCEP-MetS according to the serum calcium (The linearity for men and women is $<0.001$ after adjustment for alcohol intake, physical activity, age, smoking, and vitamin D intake). (B) Sensitivity and specificity curves

according to the range of serum calcium values separating subjects with and without MetS.
As the use of diuretics may influence serum calcium levels, we excluded all patients on the hypertensive medication $(n=1046)$, and the results remained conceptually unaltered. The frequencies of MetS according to increasing serum total calcium were 31 , 37,44 , and $54 \%(P<0.001)$ respectively. The results were in line in both sexes separately (data not shown).

\section{Discussion}

This population-based study showed that an increased serum calcium level strongly correlated with MetS, and four of five of its components in both genders (i.e. waist, $\mathrm{BP}$, fasting glucose, $2 \mathrm{~h}$ glucose, and triglycerides), even after exclusion of hypertensive patients. Increased serum calcium did not correlate with HDL-cholesterol in males or females. The threshold value of serum total calcium for MetS was $2.50 \mathrm{mmol} / \mathrm{l}$ in this population, but the areas under the ROC-curve was only 0.56 . Based on the drug treatment, hypertension, dyslipidemia, and diabetes also significantly increased in a linear trend with increasing calcium.

A large Swedish health screening survey performed from the years 1969 to 1970 found that changes in calcium metabolism, even within the physiological range, were related to hypertension, impaired glucose tolerance, and hyperlipidemia (11). Altered calcium homeostasis correlated with abnormalities of fasting serum glucose, insulin resistance, and $\beta$-cell function in the Newfoundland population study, Canada. Interestingly, this relationship remained even after adjustment for 25-OH vitamin D and PTH (12).

According to a large systematic review, dietary calcium intake or calcium supplements have only shown minimal, if any, cardiovascular effects (13). The association between vitamin D status and cardiometabolic outcomes is also uncertain. Indeed, 13 observational studies and 18 trials on cardiometabolic outcomes failed to show a clinically significant effect of vitamin D supplementation (14). In the National Health and Nutrition Examination Survey, however, low levels of vitamin $\mathrm{D}$ were associated with higher A1c values in a large non-diabetic adult population (15). Low levels of vitamin D have also been shown to be associated with insulin resistance $(16,17)$ and MetS $(18)$.

Low vitamin D levels stimulate the secretion of PTH, which is a key regulator of mineral metabolism, the homeostasis of calcium, phosphate, vitamin $\mathrm{D}$, and bone turnover. In the Uppsala, Sweden, community-based sample of elderly men, higher plasma levels of PTH were associated with a higher risk for cardiovascular mortality. This association was independent of established cardiovascular risk factors and factors associated with mineral homeostasis in the range of normal serum calcium $(2.2-2.6 \mathrm{mmol} / \mathrm{l})(19)$. Calcium is a versatile intracellular messenger that is used throughout the life cycle of an organism to control diverse biological processes (20). It 
Table 2 The drug treatment for hypertension, dyslipidemia, and diabetes according to the serum total calcium in both genders. Data are presented as $n(\%)$.

\begin{tabular}{|c|c|c|c|c|c|}
\hline \multirow[b]{2}{*}{ Drug treatment } & \multicolumn{4}{|c|}{ Serum calcium $(\mathrm{mmol} / \mathrm{l})$} & \multirow[b]{2}{*}{$P$ value } \\
\hline & $\leq 2.39$ & $2.40-2.49$ & $2.50-2.59$ & $\geq 2.60$ & \\
\hline \multicolumn{6}{|l|}{ Men } \\
\hline Hypertension & $54(35)$ & $196(32)$ & $176(36)$ & $52(54)$ & 0.005 \\
\hline Dyslipidemia & 33 (21) & $143(24)$ & $122(25)$ & 35 (36) & 0.023 \\
\hline Diabetes & $5(3)$ & $44(7)$ & $43(9)$ & $14(14)$ & 0.003 \\
\hline \multicolumn{6}{|l|}{ Women } \\
\hline Hypertension & $39(21)$ & $194(31)$ & $195(36)$ & $70(51)$ & $<0.001$ \\
\hline Dyslipidemia & 21 (11) & $98(16)$ & $106(20)$ & $43(31)$ & $<0.001$ \\
\hline Diabetes & $7(4)$ & $25(4)$ & $34(6)$ & $12(9)$ & 0.017 \\
\hline
\end{tabular}

*Significance test for linear trend.

has been suggested that diabetes and CVD are linked by a common defect. This defect could be divalent cation metabolism, which could include calcium (21). The same study group from Uppsala found that PTH correlated with several metabolic factors included in MetS, even within a normocalcemic population (22).

The essential question to investigate is why subjects with MetS have elevated serum calcium levels. Interestingly, the dietary intake of calcium does not appear to differ between individuals with MetS and healthy controls in our study population. However, some studies found that intakes of calcium and dairy products may be associated with lower prevalence of MetS in middle-aged and older women (23). The suggested negative effect of calcium supply without coadministered vitamin D on CVDs, especially myocardial infarction and stroke, is interesting and needs further studies $(24,25)$. The elevated serum calcium levels in individuals with MetS may be the result of elevated intestinal absorption, increased skeletal resorption, renal absorption, or a combination of these factors. All these potential defects are mediated by PTH levels; thus, it is possible that hyperinsulinemia and/or insulin resistance are the key determinants in disturbed PTH-regulation.

A limitation of our study was that we did not measure ionized calcium, but total serum calcium, which might be affected by serum albumin and $\mathrm{pH}$. A recent smaller study from Korea found an association between MetS and serum albumin-corrected calcium levels, and the association was unchanged after adjustment for 25(OH)D and PTH levels (26). This study consists of a larger population-based cohort with people who did not have acute diseases. Therefore, it is unlikely that deviations in serum albumin seen commonly in clinical study populations would explain these findings. We did not know $25(\mathrm{OH}) \mathrm{D}_{3}$-vitamin levels, but we measured the daily dietary intake of calcium and vitamin $\mathrm{D}$. According to literature reviews $(13,14), 25(\mathrm{OH}) \mathrm{D}_{3}$ vitamin levels do not play a crucial role in determining serum calcium levels. In our study population, the vitamin D intake was lower than the Finnish National recommendation of $7.5 \mu \mathrm{g} /$ day for $26 \%$ of the men and $46 \%$ of the women. It is known that the vitamin D status is generally low among Finnish people living at the northern altitudes. Because a relative deficiency of vitamin $\mathrm{D}$ predisposes an individual to low serum calcium levels, it is unlikely that vitamin D levels explain our findings - linking high calcium concentrations to MetS. The correlation between the prevalence of MetS and serum calcium levels was also linear after adjustment for dietary vitamin D intake. Furthermore, obese people require more vitamin $\mathrm{D}$ than their leaner counterparts to achieve recommended serum $25(\mathrm{OH}) \mathrm{D}_{3}$ levels (27). Thus, if obesity was associated with any form of vitamin $\mathrm{D}$, it would be associated with low $25(\mathrm{OH}) \mathrm{D}_{3}$ levels. Further limitation of our study is also that calculated calcium intake was solely based on dietary intake. We lack the data on the possible use of the calcium supplements.

The strength of our study was the large populationbased approach randomly selected from three different areas in Finland. Our study population also included both men and women, but the limitation is a crosssectional nature of the study.

\section{Conclusions}

The data of this study suggested that serum calcium levels were involved in all components of MetS, except HDLcholesterol. MetS should be taken into consideration when finding and interpreting serum calcium levels.

\section{Declaration of interest}

The authors declare that there is no conflict of interest that could be perceived as prejudicing the impartiality of the research reported.

\section{Funding}

The FIN-D2D study has been financially supported by the hospital districts of Pirkanmaa, South Ostrobothnia, and Central Finland, the Finnish National Public Health Institute (current National Institute for Health and Welfare), the Finnish Diabetes Association, the Ministry of Social Affairs and Health in Finland, and the Academy of Finland (grant number 129293). The dietary part has been supported by the Academy of Finland (grant number 118065 and 136895), Commission of the European Communities, Directorate C-Public Health (grant agreement no. 2004310) and Finland's Slottery Machine Association 
in cooperation with the FIN-D2D Study Group, and the Steering Committee: Pihlajamäki J, Huttunen J, Kesäniemi A, Kiuru S, Niskanen L, Oksa H, Puolakka J, Puska P, Saaristo T, Vanhala M, and Uusitupa M.

\section{Author contribution statement}

J Saltevo researched data, wrote the manuscript, contributed to the discussion, and reviewed the manuscript. L Niskanen contributed to the discussion and reviewed the manuscript. H Kautiainen researched data and edited the manuscript. J Teittinen researched data. H Oksa researched data. E Korpi-Hyövälti researched data. J Sundvall researched data and reviewed the manuscript. S Männistö researched data and reviewed the manuscript. M Peltonen researched data and reviewed the manuscript. P Mäntyselkä contributed to discussion reviewed the manuscript. M Vanhala researched data, contributed to discussion, and reviewed the manuscript.

\section{References}

1 Isomaa B, Almgren P, Tuomi T, Forsen B, Lahti K, Nissen M, Taskinen MR \& Groop L. Cardiovascular morbidity and mortality associated with the metabolic syndrome. Diabetes Care $20012 \mathbf{2 4}$ 683-689. (doi:10.2337/diacare.24.4.683)

2 Laaksonen DE, Lakka HM, Niskanen LK, Kaplan GA, Salonen JT \& Lakka TA. Metabolic syndrome and development of diabetes mellitus: application and validation of recently suggested definitions of the metabolic syndrome in a prospective cohort study. American Journal of Epidemiology 2002156 1070-1077. (doi:10.1093/aje/kwf145)

3 Sorva A \& Tilvis RS. Low serum ionized to total calcium ratio: association with geriatric diabetes mellitus and with other cardiovascular risk factors? Gerontology $1990 \quad 36 \quad 212-216$. (doi:10.1159/000213202)

4 Hagström E, Hellman P, Lundgren E, Lind L \& Ärnlöv J. Serum calcium is independently associated with insulin sensitivity measured with euglycemic-hyperinsulinaemic clamp in a community-based cohort. Diabetologia 200750 317-324. (doi:10. 1007/s00125-006-0532-9)

5 Taylor WH \& Khaleeli AA. Coincident diabetes mellitus and primary hyperthyreoidism. Diabetes/Metabolism Research and Reviews 200117 175-180. (doi:10.1002/dmrr.199)

6 Fardella C \& Rodriguez-Portales JA. Intracellular calcium and blood pressure: comparison between primary hyperparathyroidism and essential hypertension. Journal of Endocrinological Investigation $1995 \mathbf{1 8} 827-832$.

7 Begum N, Leitner W, Reusch JE, Sussman KE \& Draznin B. GLUT-4 phosphorylation and its intrinsic activity. Mechanism of $\mathrm{Ca}(2+)$ induced inhibition of insulin stimulated glucose transport. Journal of Biological Chemistry $19932683352-3356$.

8 Håglin L, Törnkvist B \& Bäckman L. Prediction of all-cause mortality in a patient population with hypertension and type 2 DM by using traditional risk factors and serum phosphate, -calcium and -magnesium. Acta Diabetologica 200744 138-143. (doi:10. 1007/s00592-007-0254-6)

9 Männistö S, Virtanen M, Mikkonen T \& Pietinen P. Reproducibility and validity of a food frequency questionnaire in a case-control study on breast cancer. Journal of Clinical Epidemiology 199649 401-409. (doi:10.1016/0895-4356(95)00551-X)

10 Grundy SM, Cleeman JI, Merz CN, Brewer HB Jr, Clark LT, Hunninghake DB, Pasternak RC, Smith SC Jr \& Stone NJ \& Coordinating Committee of the National Cholesterol Education Program. Implications of recent clinical trials for the National Cholesterol Education Program Adult Treatment Panel III Guidelines. Journal of the American College of Cardiology $2004 \mathbf{4 4}$ 730-732. (doi:10.1016/j.jacc.2004.07.001)

11 Lind L, Jakobsson S, Lithell H, Wengle B \& Ljunghall S. Relation of serum calcium concentrations to metabolic risk factors for cardiovascular disease. BMJ 1988 297 960-963. (doi:10.1136/ bmj.297.6654.960)
12 Sun G, Vasdev S, Martin GR, Gadag V \& Zhang H. Altered calcium homeostasis is correlated with abnormalities of fasting serum glucose, insulin resistance, and b-cell function in the Newfoundland population. Diabetes $2005 \mathbf{5 4} 3336-3339$. (doi:10.2337/ diabetes.54.11.3336)

13 Wang L, Manson JE, Song Y \& Sesso HD. Systematic review: vitamin $\mathrm{D}$ and calcium supplementation in prevention of cardiovascular events. Annals of Internal Medicine 2010152 315-323.

14 Pittas AG, Chung M, Trikalinos T, Mitri J, Brendel M, Patel K, Lichtenstein AH, Lau J \& Balk EM. Systematic review: vitamin D and cardiometabolic outcomes. Annals of Internal Medicine 2010 152 307-314.

15 Kositsawat J, Freeman VL, Gerber BS \& Geraci SC. Association of A1c levels with vitamin D status in U.S. adults. Diabetes Care 2010 33 1236-1238. (doi:10.2337/dc09-2150)

16 Pinelli NR, Jaber LA, Brown MB \& Herman WH. Serum 25-hydroxy vitamin D and insulin resistance, metabolic syndrome, and glucose intolerance among Arab Americans. Diabetes Care 201033 1373-1375. (doi:10.2337/dc09-2199)

17 Kayaniyil S, Vieth R, Retnakaran R, Knight JA, Qi Y, Gerstein HC, Perkins BA, Harris SB, Zinman B \& Hanley AJ. Association of vitamin $D$ with insulin resistance and $\beta$-cell dysfunction in subjects at risk for type 2 diabetes. Diabetes Care 201033 1379-1381. (doi:10.2337/dc09-2321)

18 Ford ES, Ajani UA, McGuire LC \& Liu S. Concentrations of serum vitamin $\mathrm{D}$ and the metabolic syndrome among U.S. adults. Diabetes Care 200528 1228-1230. (doi:10.2337/diacare.28.5.1228)

19 Hagström E, Hellman P, Larsson TE, Ingelsson E, Berglund L, Sundström J, Melhus H, Held C, Lind L, Michaelsson K \& Ärnlöv J. Plasma parathyroid hormone and the risk of cardiovascular mortality in the community. Circulation $20091192765-2771$. (doi:10.1161/CIRCULATIONAHA.108.808733)

20 Berridge MJ, Lipp P \& Bootman MD. The versatility and universality of calcium signaling. Nature Reviews. Molecular Cell Biology 2000 1 11-21. (doi:10.1038/35036035)

21 Resnick LM. Hypertension and abnormal glucose homeostasis: possible role of divalent ion metabolism. American Journal of Medicine 198987 17S-22S. (doi:10.1016/0002-9343(89)90490-7)

22 Ahlström T, Hagström E, Larsson A, Rudberg C, Lind L \& Hellman P. Correlation between plasma calcium, parathyroid hormone (PTH) and the metabolic syndrome (MetS) in a community-based cohort of men and women. Clinical Endocrinology 200971 673-678. (doi:10.1111/j.1365-2265.2009.03558.x)

23 Liu S, Song Y, Ford ES, Manson JAE, Buring JE \& Ridker PM. Dietary calcium, vitamin D, and the prevalence of metabolic syndrome in middle-aged and older U.S. women. Diabetes Care 200528 2926-2932. (doi:10.2337/diacare.28.12.2926)

24 Bolland MJ, Avenell A, Baron JA, Grey A, MacLennan GS, Gamble GD \& Reid IR. Effect of calcium supplements on risk of myocardial infarction and cardiovascular events: meta-analysis. BMJ 2010341 c3691 (doi:10.1136/bmj.c3691). (doi:10.1136/ bmj.c3691)

25 Bolland MJ, Grey A, Avenell A, Gamble GD \& Reid IR. Calcium supplements with or without vitamin D and risk of cardiovascular events: reanalysis of the Women's Health Initiative limited access dataset and meta-analysis. BMJ $2011 \mathbf{3 4 2}$ d2040 (doi:10.116/bmj.d2040). (doi:10.1136/bmj.d2040)

26 Kim MK, Kim G, Jang EH, Kwon HS, Baek KH, Oh KW, Lee JH, Yoon KH, Lee WC, Lee KW, Son HY \& Kang MI. Altered calcium homeostasis is correlated with the presence of metabolic syndrome and diabetes in middle-aged and elderly Korean subjects: the Chungju Metabolic Disease Cohort study. Atherosclerosis 2010 212 674-681. (doi:10.1016/j.atherosclerosis.2010.07.005)

27 Lagunova Z, Porojnicu AC, Lindberg F, Hexeberg S \& Moan J. The dependency of vitamin D status on body mass index, gender, age and season. Anticancer Research $2009293712-3720$.

Received 20 May 2011

Accepted 9 June 2011 